\title{
Enhanced Bragg reflections from size-matched heterostructure photonic crystal thin films prepared by the Langmuir-Blodgett method
}

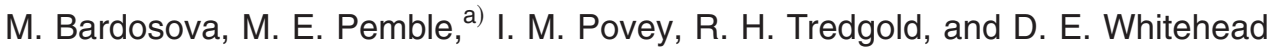 \\ Tyndall National Institute, University College Cork, Lee Maltings, Prospect Row, Cork, Ireland (Republic)
}

(Received 10 March 2006; accepted 7 July 2006; published online 30 August 2006)

\begin{abstract}
The Langmuir-Blodgett method was used to engineer photonic crystal thin films of an $\mathrm{AB}$ architecture. Structures were studied by transmittance and reflectance spectroscopies. For an AB structure in which the silica particle diameter B is twice that of A, reflectance features associated with the first order Bragg peak for the "A" domain are only observed when the structure is probed from the A side of the structure. Furthermore, this feature is enhanced in intensity compared to that for a structure consisting solely of A particles. These findings are attributed to a matching of first and second order Bragg processes. (C) 2006 American Institute of Physics. [DOI: 10.1063/1.2339031]
\end{abstract}

Recently there has been considerable interest in the formation of films of synthetic opals because of their possible application in photonics. ${ }^{1-3}$ The fabrication of multilayer systems using particles of differing diameters has been studied previously for both controlled evaporation ${ }^{4-6}$ and the Langmuir-Blodgett (LB) method. ${ }^{7,8}$ Optical studies on multilayer systems have to date focused on transmission spectra to define their optical properties. Rengarajan et al. ${ }^{4}$ utilized transmission spectroscopy to detect midgap states engineered within a multilayer photonic crystal. Jiang et al. ${ }^{5}$ studied multilayer systems of particles with overlapping optical responses to observe a broadened stop band within the transmission spectra. Binary Langmuir-Blodgett composite crystals have recently been studied by Reculusa et $a l^{8}$ and Masse and Ravaine, ${ }^{9}$ however, the particles in these studies did not have a diameter ratio of 2 and the spectra were only measured in transmission and would thus not be expected to exhibit the phenomenon which we report.

In our present work we have examined three binary structures of ten layers of one particle size deposited on a glass substrate followed by ten layers of a different particle size. The three particle diameters are $200 \mathrm{~nm}$ (A), $400 \mathrm{~nm}$ (B), and $300 \mathrm{~nm}(\mathrm{C})$; of these we concentrate on the $\mathrm{AB}$ structure with $\mathrm{BA}$ and $\mathrm{AC}$ structures being used to highlight the properties of the $\mathrm{AB}$ system. The resultant films were studied optically in both transmission and reflectance modes. We demonstrate that, in contrast to previous studies where only transmission measurements have been made, the use of reflectance measurements reveals a property of the sizematched $(\mathrm{AB})$ heterostrucutre photonic crystal films that has not been previously reported.

Silica particles were fabricated using the well-known Stöber ${ }^{10}$ technique and made hydrophobic by coating with 3-(trimethoxysilyl)propyl methacrylate. The methods of particle treatment and LB film deposition have been described previously, ${ }^{11}$ with the key parameters being a substrate withdrawal speed of $0.5 \mathrm{~cm} \mathrm{~min}^{-1}$, surface pressures between 3 and $6 \mathrm{mN} \mathrm{m}^{-1}$, and a barrier speed equivalent to $6 \mathrm{~cm}^{2} \min ^{-1}$.

For a face centered cubic (fcc) lattice, where the [111] direction is normal to the surface of the substrate, it can be

\footnotetext{
${ }^{a)}$ Electronic mail: martyn.pemble@tyndall.ie
}

shown that the first order maximum wavelength of the diffracted wave is given by

$$
\lambda=2(2 / 3)^{1 / 2} D\left(n^{2}-\sin ^{2} \theta\right)^{1 / 2},
$$

where $n$ is the mean refractive index of the film, $D$ the diameter of the spherical particles, and $\theta$ is the angle between the normal to the surface and the incident and diffracted waves. Thus by plotting $\lambda^{2}$ vs $\sin ^{2} \theta$ it is possible to obtain the value of $D$. It should be noted that, in the angular region of $45^{\circ}$ for a fcc lattice, diffraction from the (200) plane also appears and thus the expression given above is no longer correct and a double diffraction peak appears. This phenomenon has been explained by Romanov et al. ${ }^{12}$ However, for films formed by the LB method this double peak does not appear and the apparent diameter of the particles derived from the Bragg expression is somewhat larger than the value obtained by other methods showing that although an ordered structure appears it is not fcc. This feature of the LB growth of photonic crystals from colloidal particles will be discussed in detail elsewhere. However, it is clear that each individual plane of particles is well ordered. As a consequence the actual diameter of particles employed in this work was derived independently using Eq. (1) in conjunction with films obtained by a controlled evaporation assembly method, which does produce a fcc lattice.

In Fig. 1(a) we show the spectra obtained by transmission at normal incidence for films formed from $400 \mathrm{~nm}$ (B) particles deposited on top of the $200 \mathrm{~nm}$ (A) particles (AB film) and in Fig. 1(b) the structure obtained by depositing the $200 \mathrm{~nm}$ (A) particles on top of the $400 \mathrm{~nm}$ (B) particles (BA film). Similar spectra were obtained from both specimens irrespective of which direction they were illuminated. It should be noted that some splitting is observed in the Bragg peak of the BA structure, which is not evident in that of the AB. The splitting in the spectrum of the BA structure is attributed to interface effects, as observed by Rengarajan et $a l^{4}$ and is further complicated in appearance by overlapping Fabry-Pérot oscillations. The interface effects can be thought as arising due to the layer containing the smaller particles "sagging" into the hollows formed by the layer of larger particles, hence distorting the structure at the interface via the superposition of a periodicity on a larger scale. Correspondingly the absence of splitting in the Bragg peak of the $\mathrm{AB}$ structure can be explained by the two crystal regions 

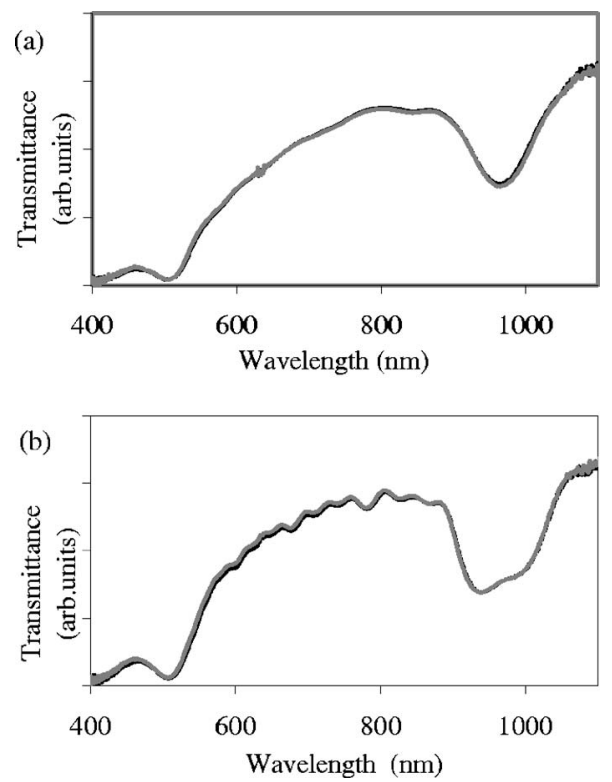

FIG. 1. Transmission spectra of (a) the AB structure and (b) the BA structure taken from both the A and B surfaces. As would be predicted the spectra are not dependent on the direction of illumination.

remaining distinct as the integrity of the large particle interface layer is easier to maintain when the particles below are smaller, an observation that is supported by the scanning electron microscopy (SEM) images of controlled evaporation multilayer systems produced by Wong et al. ${ }^{6}$ and by images of the layers produced in this present work (Fig. 2). From Fig. 2 it may be seen that the presence of interface effects alluded to earlier is supported by the observation that for the $\mathrm{AB}$ structure [Fig. 2(a)], the interface between the A and B domains appears clearly defined and sharp, whereas for the BA structure [Fig. 2(b)], the SEM images show clear evidence of the A layers sagging into the voids present in the B layer.

Figure 3 depicts reflectance spectra obtained at an angle of $12^{\circ}$ from normal incidence for (a) the $\mathrm{AB}$ structure and (b) the BA structure. In each case spectra have been recorded

(a)

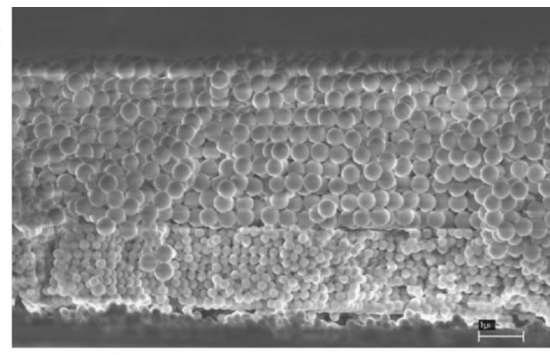

(b)

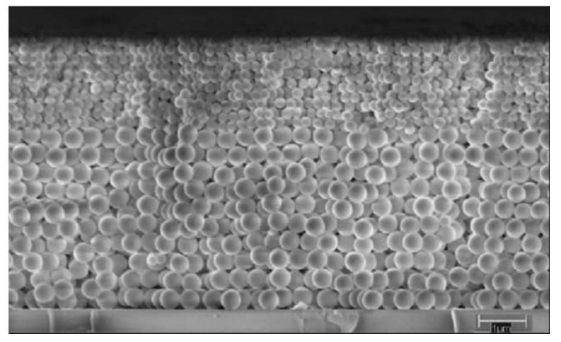

FIG. 2. SEM images of cleaved cross sections of the AB and BA structures. The interface in the $A B$ structure (a) is clearly defined whereas that for the BA structure (b) shows evidence of sagging within the B layer.

Downloaded 15 Sep 2006 to 143.23965 .96. Redistribution subject
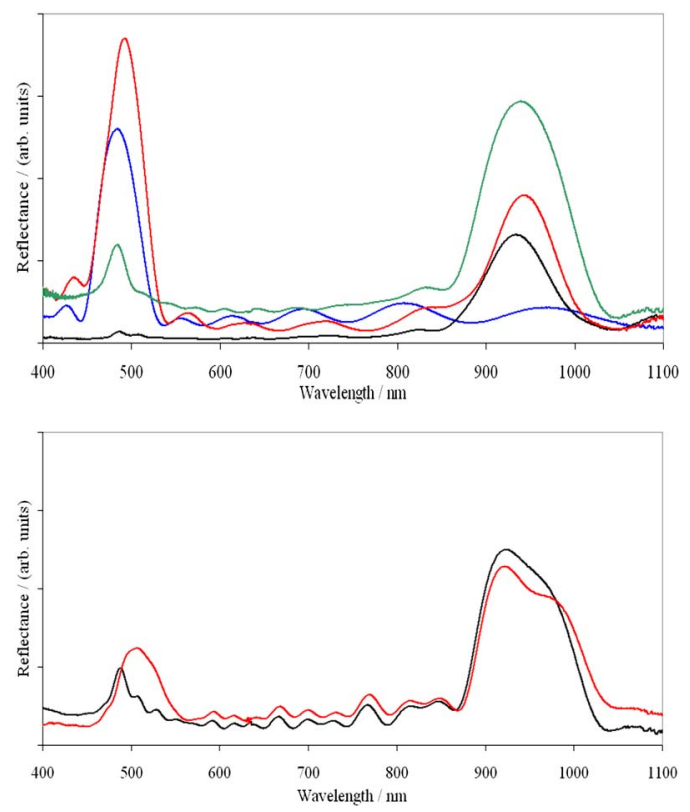

FIG. 3. (a) Reflection spectra of the AB structure taken from the A surface (red) and B surface (black). The reflection spectra of comparable ten layer A only (blue) and ten layer B only (green) crystals are given for comparison. The intensity of the $\sim 500 \mathrm{~nm}$ peak is clearly enhanced when illuminated from the A surface. (b) Reflection spectra of the BA structure taken from the A surface (red) and B surface (black). Enhancement is not observed and the spectra are complicated by an interface effect.

from both the top of the samples (red lines) and the bottom, i.e., through the glass substrate (black lines). Figure 3(a) also depicts the reflectance spectrum obtained from films of ten layers of type A particles (blue line) and B particles (green line) for direct comparison with the $\mathrm{AB}$ film.

From Fig. 3 it is apparent that the peak at ca. $500 \mathrm{~nm}$ corresponding to the first order Bragg reflection from the type A particle layer under the type B particle layer is very intense for the case when the sample is probed through the underlying glass substrate, yet very weak when the sample is probed from the top, through the type B particle layer. Of particular note is that the peak intensity when the $\mathrm{AB}$ film is probed from the bottom side is greater than that for the film containing only type A particles (blue line). The possible inference that this intensity enhancement arises from a substrate effect is negated by comparison with the BA system [Fig. 3(b)], where illuminating from below yields a spectrum, that is comparable to that obtained by illuminating from above. For this reason the passage of the light through the glass substrate cannot in itself explain the intensity anomaly observed for the $\mathrm{AB}$ structure.

We propose that these results arise from the fact that the illumination of the $\mathrm{AB}$ structure from the top with a wavelength of ca. $500 \mathrm{~nm}$ (corresponding to a situation where normally there would be a first order reflection from the layers of type A particles having a diameter of $200 \mathrm{~nm}$ ) results in the amplitude of the incident light being severely attenuated by first passing through the region of type B particles having a diameter of $400 \mathrm{~nm}$, which themselves generate a strong first order Bragg reflection at about $960 \mathrm{~nm}$. The weak nature of the second order Bragg reflection of the B layer, at the wavelength where the layer of type A particles will have a first order Bragg peak, i.e., ca. $500 \mathrm{~nm}$, leads to the type B layer severely attenuating the light at this wavelength reaching the A layer and thus little or no first order Bragg peak AlP license or copyright, see http://apl.aip.org/apl/copyright.jsp 
from the type A particle layer will be seen. The assumption that this is an attenuation effect by the B particle layer is supported by our observation that the reflection spectrum, at this wavelength, is attenuated as a function of the B layer thickness. In contrast when the $\mathrm{AB}$ sample is illuminated from the bottom, such that the type A particle layer sees the light first with no prior attenuation, the first order Bragg peak from the type A particle layer is very intense and enhanced by the overlap with the weaker second order peak from the type B particle layer, i.e., an additive process occurs.

Similar behavior might be expected to occur for the BA structure, in that some enhancement of the first order Bragg peak for the type A particle layer might be expected, this time under illumination from the top. Figure 3(b) reveals that while this peak is certainly more intense than its counterpart recorded via illumination from the bottom, it is much weaker than the enhanced peak depicted in Fig. 3(a). We attribute this observation to the disruption of the quality of the photonic crystal formed from the BA structure due to the interface effects referred to earlier.

In order to confirm this hypothesis a third structure, denoted AC, consisting of ten layers of particles having a diameter of $200 \mathrm{~nm}$ (A) on top of which were ten layers of particles having a diameter of $300 \mathrm{~nm}$ (C) was studied. It was found that in this case where there is no size matching, the spectra obtained could be interpreted purely on the basis of light attenuation due to scattering and that the intensity of the Bragg peak for the underlying type A particle layer did not show any unexpected variation in intensity. When illuminated from the bottom (type A particle layer first), the intensity of the corresponding Bragg peak was greater than for the case where the sample was illuminated from the top (type $\mathrm{C}$ particle layer first) by a factor of ca. 2, as might be expected if one simply considers the likely losses involved.
To conclude we believe that we have observed the strong effect of second order Bragg diffraction in a composite heterostructure opal film consisting of size-matched particles. Importantly the dramatic differences in the optical properties for the $\mathrm{AB}$ and $\mathrm{BA}$ films reported here can only be seen in reflectance. Results suggest that the additive effects of the first order Bragg reflection from the type A particle layer and the second order Bragg reflection from the type B particle layer work most efficiently for the $\mathrm{AB}$ structure as compared to the BA, due to the occurrence of interface effects in the latter structure which disrupts the order.

Thus the principle finding presented here is that in reflection mode, size-matched heterostrucutre photonic crystals may exhibit cooperative effects whereby the first order Bragg reflections from one part of the structure mix with the second order Bragg reflections from other parts of the structure. We suggest that the resulting differences in reflectance for the $\mathrm{AB}$ samples for the different modes of illumination may find exploitation in a range of optical filtration devices.

${ }^{1}$ V. L. Colvin, MRS Bull. 26, 637 (2001).

${ }^{2}$ M. Bardosova and R. H. Tredgold, J. Mater. Chem. 12, 2835 (2002).

${ }^{3}$ C. Lopez, Adv. Mater. (Weinheim, Ger.) 15, 1679 (2003).

${ }^{4}$ R. Rengarajan, P. Jiang, D. C. Larrabee, V. L. Colvin, and D. M. Mittleman, Phys. Rev. B 64, 205103 (2001).

${ }^{5}$ P. Jiang, G. N. Ostojic, R. Narat, D. M. Mittleman, and V. L. Colvin, Adv. Mater. (Weinheim, Ger.) 13, 389 (2001).

${ }^{6}$ S. Wong, V. Kitav, and G. A. Ozin, J. Am. Chem. Soc. 125, 15589 (2003).

${ }^{7}$ S. Reculusa and S. Ravaine, Chem. Mater. 15, 598 (2003).

${ }^{8}$ S. Reculusa, P. Masse, and S. Ravaine, J. Colloid Interface Sci. 279, 471 (2004).

${ }^{9}$ P. Masse and S. Ravaine, Chem. Mater. 17, 4244 (2005).

${ }^{10}$ W. Stöber, A. Fink, and E. Bohn, J. Colloid Interface Sci. 26, 62 (1968).

${ }^{11}$ M. Bardosova, P. Hodge, L. Pach, M. E. Pemble, V. Smatko, R. H. Tredgold, and D. Whitehead, Thin Solid Films 437, 276 (2003).

${ }^{12}$ S. G. Romanov, T. Maka, C. M. Sotomayor Torres, M. Muller, R. Zentel, D. Cassagne, J. Manzanares, and C. Jouanin, Phys. Rev. E 63, 56603 (2001). 